\title{
Industrial-Strength Documentation for ACL2
}

\author{
Jared Davis \\ Formal Verification Group \\ Centaur Technology \\ jared@centtech.com
}

\author{
Matt Kaufmann \\ Department of Computer Science \\ University of Texas at Austin \\ kaufmann@cs.utexas.edu
}

\begin{abstract}
The ACL2 theorem prover is a complex system. Its libraries are vast. Industrial verification efforts may extend this base with hundreds of thousands of lines of additional modeling tools, specifications, and proof scripts. High quality documentation is vital for teams that are working together on projects of this scale. We have developed XDOC, a flexible, scalable documentation tool for ACL2 that can incorporate the documentation for ACL2 itself, the Community Books, and an organization's internal formal verification projects, and which has many features that help to keep the resulting manuals up to date. Using this tool, we have produced a comprehensive, publicly available ACL2+Books Manual that brings better documentation to all ACL2 users. We have also developed an extended manual for use within Centaur Technology that extends the public manual to cover Centaur's internal books. We expect that other organizations using ACL2 will wish to develop similarly extended manuals.
\end{abstract}

\section{Introduction}

Since its earliest versions, the ACL2 theorem prover has featured a comprehensive user's manual. You can find references to this documentation even as far back as the release notes for ACL2 version 1.1, dating from October 1990. This documentation was initially available only from the terminal, but by 1994, Kaufmann and Moore [7] reported that it had grown to feature a markup language and conversion tools for producing manuals:

"The ACL2 documentation is maintained in a hypertext-like structure which may be browsed via ACL2 documentation commands. In addition, it may be browsed via Emacs' Info and via Mosaic. Roughly .9 megabytes of online documentation is available about ACL2, including tutorials. Instructional materials are being prepared as part of the documentation. The ACL2 user may wish to document his or her formal models and browse that documentation with the facilities provided..."

This mention of Mosaic is amusing, but lends some historical context. Milestones like the first draft specification of HTML and the first alpha of Mosaic had each occurred only about a year earlier. Browsers like Netscape were not yet available.

This documentation system-now called the legacy documentation system - went on to serve, largely unchanged, as the basis for the ACL2 User's Manual for the next twenty years. This time period spanned dozens of ACL2 releases and thousands of changes to ACL2. Throughout these changes, great care was taken by Kaufmann and Moore to expand the manual and keep its contents up to date; the manual tripled in size from ACL2 Version 1.9 in 1994, the earliest public release, through Version 6.3 in 2013.

But meanwhile, as web technologies advanced, the presentation, navigation, and organization of the manual were not the focus. In Figure 1, you can see the great similarity between the key navigation pages from Versions 1.9 and 6.3. By way of contrast, Figure 2 shows manuals produced by the Javadoc and Doxygen tools, which, for instance, take advantage of frame-based navigation.

F. Verbeek and J. Schmaltz (Eds.): ACL2 Workshop 2014 (ACL2'14). EPTCS 152, 2014, pp. 9-25, doi:10.4204/EPTCS.152.2 (c) Jared Davis and Matt Kaufmann

This work is licensed under the Creative Commons Attribution License. 

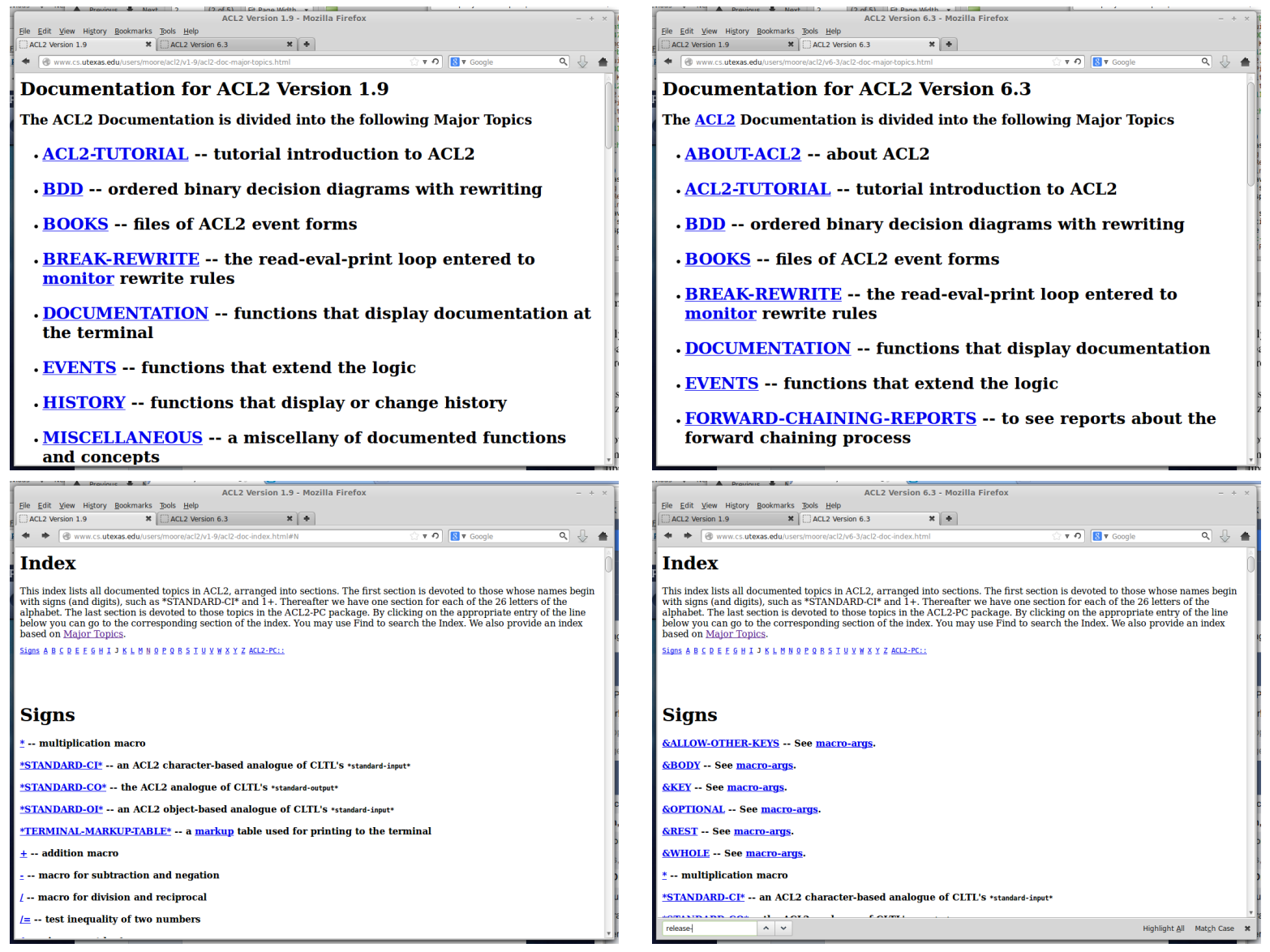

Figure 1: ACL2 User Manual navigation pages for versions 1.9 (left) and 6.3 (right).


Figure 2: Java API manual from Javadoc (left) and GNU Radio manual from Doxygen (right). 
Moreover, throughout this time, the manual only covered the functionality provided by the theorem prover itself. While many topics mentioned related Community Books and workshop papers, there were no direct links. Even well-established libraries like data-structures and ihs, which had documentation, were excluded from the manual. The tools for creating manuals for books were difficult to use and had various problems with packages. Jared Davis described these and other frustrations with the legacy documentation system in a September 3, 2009 email to Matt Kaufmann and Alan Dunn. He explained his motivation as follows:

"At Centaur we are developing a lot of books, and we are making use of lots of books in : dir : system. We have a number of users, with varying levels of ACL2 proficiency, and varying levels of understanding of each library. In some cases they may not even know what libraries they are using. Because of this, it would be really nice to be able to point them to documentation for our whole system, instead of just pieces of it... [This] does not work."

Dunn was able to quickly patch the legacy documentation system to correct these package problems, allowing Centaur to use it, in the short term, in their X86 processor verification efforts. But to address the deeper issues, we began working on a new documentation system for ACL2 named XDOC. On October 20, we committed a preliminary version to the Community Books repository. In the years since, we have grown XDOC into a mature and capable documentation tool for ACL2.

This paper is a broad introduction to XDOC for the ACL2 community. It provides some historical context and describes our motivation for developing a new documentation system. It explains how XDOC impacts the ACL2 User's Manual, and the new options for viewing ACL2's documentation. It shows how you can use XDOC to document your own books, and how to do this in elegant ways that can reduce the burden of writing and maintaining documentation. It also describes how organizations can effectively use XDOC to develop comprehensive manuals for their verification projects.

Just what is XDOC? Like many software documentation tools, it allows pieces of documentationdescribing a function, a theorem, or what have you - to be scattered throughout the source code of many books. This documentation can make use of an XML-based markup language and also a preprocessor that has a number of features. We describe the basics of writing XDOC documentation in Section 2.

XDOC can assemble these disparate topics to create a manual for a fancy web-based viewer or for an Emacs-based viewer, both shown in Figure 3. These manuals can be very comprehensive. Starting
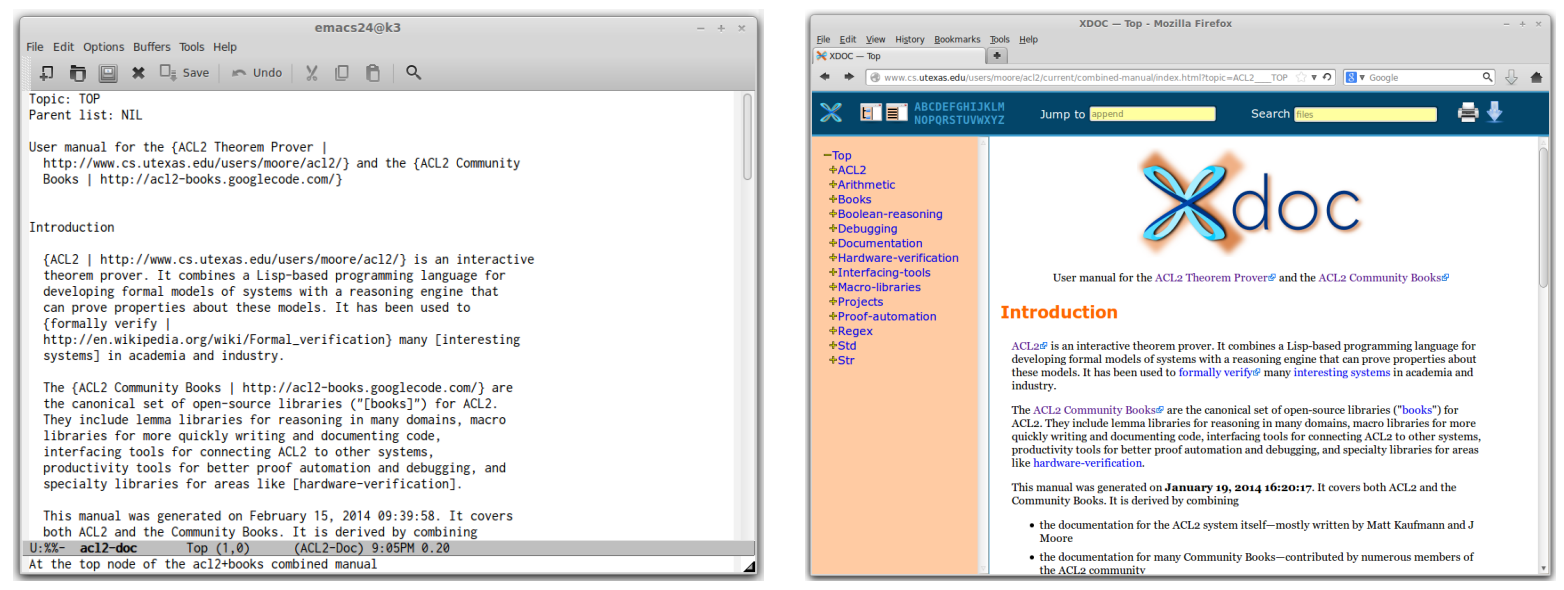

Figure 3: XDOC manuals in the Emacs Viewer (left) and the Fancy Viewer (right). 
with ACL2 version 6.4, we have converted the documentation for ACL2 itself from its legacy format into XDOC format. Meanwhile, over the past few years, many Community Books have been extended with XDOC documentation. In Section 3, we explain how these efforts have allowed us to create a single, integrated ACL2+Books Manual that covers both ACL2 itself and a large collection of Community Books. We also describe the options for accessing this manual, and explain how it can be extended to cover additional books.

For industrial verification projects, XDOC can also create extended manuals that cover additional books that are internal to an organization. For instance, the formal verification group at Centaur Technology now has a custom manual that extends the ACL2+Books Manual with thousands of additional topics that are specific to their particular modeling and verification efforts. This manual is built nightly and is available company-wide as an intranet web page. David Rager has told us that a similar manual is being used internally at Oracle. We explain how to create extended manuals in Section 4.

A major reason to embed the documentation in the source code is that this makes it easier to keep the manual updated as the source code is changed. Good software documentation tools can also automatically extract and use information from the source code, e.g., function signatures, class hierarchies, etc., to provide context, generate navigation pages, and so on, which avoids duplication between the manual and the code and helps to keep the manual current. Unfortunately, features of ACL2 such as dynamic typing and macros, and also its lack of any built-in notion of module, conspire to make this kind of automatic extraction difficult. In Section 5 we explore this difficulty and describe some solutions. We have developed some basic tools for automatically grouping related events and including them in a documentation topic. We have also been able to develop documentation-aware macros that can produce high quality documentation without duplicating code. To highlight this, we show how certain macros in the std/util library are now integrated with XDOC.

\section{Writing Documentation with XDOC}

As groundwork for reading the rest of this paper, it will be very useful to have a basic feel for how XDOC documentation is written. In this section, we dissect an example topic that illustrates the basics.

Since XDOC is a library, even its most basic documentation commands are not included in ACL2 itself. The first step to using XDOC is to load it via:

(include-book "xdoc/top" :dir :system)

You may find that you already have this book loaded, as it is now widely included throughout the Community Books. If not, it loads very quickly, does not alter your theory, and is trust-tag free. Once it is loaded, we can begin writing documentation. The basic unit of documentation in XDOC is a topic, and the most primitive way to add a new topic is called defxdoc. Here is an example:

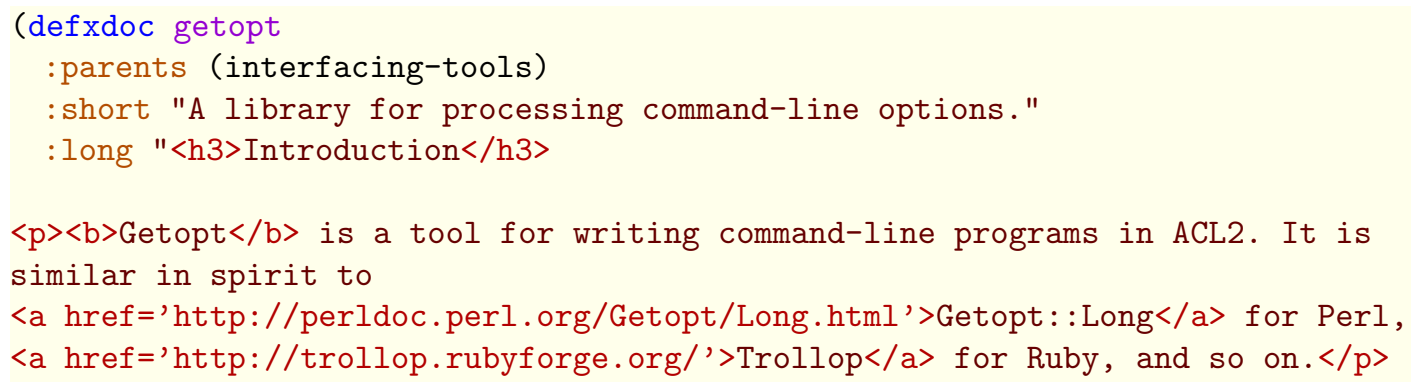


$<\mathrm{p}>$ We basically extend @(see defaggregate) with a command-line parsing layer.

This has some nice consequences: $\langle/ p\rangle \ldots "$ )

As a quick explanation:

- Each topic has a unique name which must be an ACL2 symbol-in this case getopt. Since ACL2 symbols belong to particular packages, the usual package mechanism can be used to avoid name clashes when combining documentation from many libraries.

- The parents refer to other topics that should typically cover broader or more general concepts. This parent information is used to group documentation into a hierarchy that supports basic navigation features in viewer programs. Most topics have only a single parent, but having additional parents can be useful when a topic fits well in many places. For instance, it allows the std/util library to be listed as part of the std library, and also under the macro-libraries category.

- The short and long strings, together, make up the real contents of a topic. The short string is intended to be a 1-2 sentence summary of the topic. Viewers embed it into navigational devices such as tooltips, child topic listings, search results, and so on. The long string contains the main contents of the topic. Both strings are optional, which is useful for topics that need little explanation.

The short and long strings can make use of a conventional, XML markup language with tags like $\langle\mathrm{p}\rangle,\langle\mathrm{h} 3\rangle$, etc. This language is very similar to a simple subset of HTML except that the tags must be strictly balanced. XML is very widely supported. For instance, web browsers can directly load XDOC's markup and, e.g., traverse it using Javascript, or convert it into HTML using XSLT stylesheets. More generally, almost every programming language has libraries for parsing and working with XML files.

In addition to XML tags, the short and long strings can also make use of preprocessor directives such as $@$ (see defaggregate). The preprocessor is a central feature of XDOC. It is used to translate these special @ directives into plain XML when you create a manual or display an individual topic with the : doc command. For instance, in this case it will convert $@$ (see defaggregate) into <see topic='STD___DEFAGGREGATE' $>$ def aggregate $</$ see $>$.

The preprocessor can do many things. For instance, its $@($ see ...) directive handles the details of symbol name encoding and allows topics to be referenced simply, usually without package prefixes. Its special $@\left(' \ldots{ }^{\prime}\right)$ and $@(\{\ldots\})$ syntax can be used to write raw or verbatim text without escaping XML characters like < as \&lt; , and automatically links to documented topics. For instance, the translation of $@($ ' ( $\operatorname{car}$ (append $\mathrm{x} y))$ ') will have links to the topics for car and append. Other directives can be useful for automatically generating documentation and keeping the documentation up to date. For instance, its $@(\operatorname{def} . .$.$) directive can automatically look up functions, theorems, etc., from the$ ACL2 world, and insert them into the documentation as code blocks with automatic links to any documented topics. Other directives allow for evaluating ACL2 expressions and injecting the results into the documentation, which may be useful for ensuring that documented examples are correct.

The defxdoc command is a real ACL2 event. Many other documentation tools, such as Javadoc and Doxygen, instead use special comments like $/ * * \ldots * /$ to embed the documentation into the source code. These comments can be extracted by the documentation tool and associated with nearby source code elements, but are invisible to the Java or C++ compiler. This means it isn't possible to dynamically manipulate existing documentation.

In contrast, XDOC stores all of its topics in an ordinary ACL2 table, and defxdoc just adds a new topic to this table. One consequence of this is that, when interacting with ACL2, all of the documentation for the books you have loaded and the events you have submitted is immediately available using the usual : doc command. For instance, after submitting the getopt topic above, we can display it as text: 


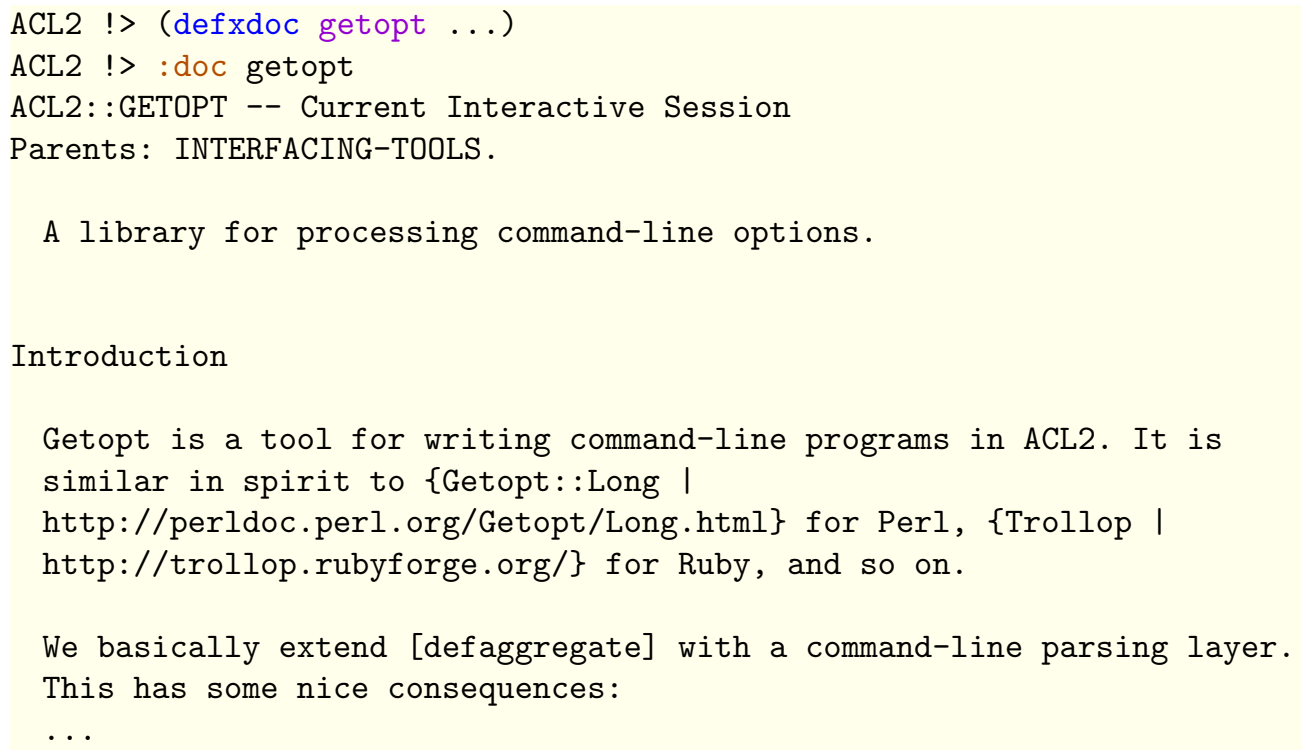

Having the documentation in a table also means that we can easily write code to extend existing topics, change parent relationships, and so forth. In Section 5, we will see some interesting and useful ways to take advantage of these capabilities.

\section{The ACL2+Books Manual}

Starting in ACL2 6.4, the documentation for ACL2 has been converted into XDOC format, made editable by the Community, and integrated with the documentation for the Community Books to create a convenient ACL2+Books Manual. In this section, we describe some of the history leading up to this change, the new options available for viewing this manual, and how members of the ACL2 Community can add new documentation to the manual.

\subsection{Steps Toward a Unified Manual}

Although the initial version of XDOC (October 2009) lacked many basic features such as navigation pages, its XML markup language and preprocessor were similar to the XDOC of today. Within a few months, we added a basic navigation system and the formal verification group at Centaur began using XDOC. Over the next year, many libraries were documented with it.

Meanwhile, ACL2 itself and many other libraries were still documented with the legacy system. Frustrated with this schism, Warren Hunt challenged us to unify the manuals. Toward this goal, we developed a tool for incorporating legacy documentation into XDOC and implemented an : xdoc command for interactive browsing. In January 2011, we published an extremely preliminary ACL2+Books Manual and announced it to the ACL2-Books email list. Over the next years, as ever more XDOC documentation became available, the convenience of a combined manual became increasingly apparent. With the release of ACL2 5.0 in August 2012, Centaur began hosting unofficial ACL2+Books Manuals.

The tipping point came in July 2013 when we developed a new, fancy web-based XDOC viewer. The new viewer included many expanded navigation options, e.g., jumping to topics by name, flat and hierarchical navigation, and the ability to expand subtopics inline within a page. With such an improved viewer, it seemed that the time to retire the legacy system had come. In September, Davis presented 
XDOC to the ACL2 Seminar at UT, and proposed converting ACL2's documentation into XDOC format and making it editable by the community to better integrate the system- and book-level documentation.

After developing some custom tools to automate the process, we carried out this conversion and developed a way to load the resulting documentation — now separate from ACL2 - back into the theorem prover at build time, so that the :doc command would continue to function even without including any of the XDOC books (which convert : doc to :xdoc using the ld-keyword-aliases feature of ACL2). One last sticking point was that XDOC did not support browsing the documentation in Emacs. We developed a new Emacs browser, named ACL2-Doc, that is easy to use, provides several useful capabilities not present in its predecessor that was based on Emacs Info, and can automatically download a gzipped copy of the ACL2+Books manual.

With the technical hurdles out of the way, on October 27, 2013 we announced the new manual to the general ACL2 mailing list. Since then, we have begun to take advantage of XDOC features in ACL2 documentation topics, and to more closely integrate the system and book documentation. For instance, we have extended topics for many simple functions like append with $@$ (def ...) directives so that the documentation shows their definitions. In many cases we have added new links from ACL2 topics into related library documentation. We have also worked to improve the organization of topics, e.g., new sections like interfacing-tools allow system-level topics like io, save-exec and sys-call to be grouped with libraries such as getopt, oslib, and quicklisp. We have also worked to move many topics out of patchwork areas like miscellaneous and other and into more germane sections.

\subsection{Viewing the Manual}

There are currently three main ways to view documentation: the interactive : doc command, the fancy web-based viewer, and the ACL2-Doc viewer for Emacs.

The : doc command works much as it always has. Although it is a text-only display that lacks, e.g., colors, clickable links, and so forth, we often find it useful for quick reference. The most important thing to note is that it will only display documentation for (1) the theorem prover itself, and (2) books that you have loaded. This has always been true, even for the legacy documentation system, but the effect is now more pronounced as so many books now have documentation.

We expect that most users will want to use the fancy web-based edition of the ACL2+Books Manual, which is available on the ACL2 web site. We plan to update this manual with each ACL2 release, as has historically been done for the ACL2 User's Manual. For users of bleeding-edge, development versions of ACL2 and the Community Books, there is also a frequently updated documentation snapshot.

While using the web-based version should be largely self-explanatory, a couple of features are worth noting. If you use Emacs, you may be able to configure your browser so that clicking on special gray Emacs links (as shown to the right) will automatically trigger a tags search — this can be very convenient, but requires some setup; for details, see the

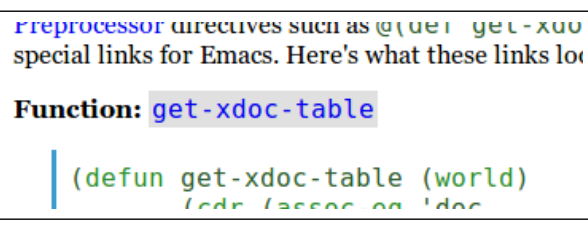
documentation on XDOC. If you have a slow or intermittent network connection, every XDOC manual includes a Download this Manual button which makes it easy to get a local copy for offline browsing. Finally, the $\boxplus$ button next to Subtopics listings can be used to expand whole sections of the manual into a single page, which can be especially useful when reading or printing tutorial-style material.

The third way to view documentation is with the ACL2-Doc Emacs browser. This view is similar to the Emacs Info version of the ACL2 User's Manual in the legacy documentation system. However, it has additional capabilities and, most importantly, it can view not only the ACL2 User's Manual but also the 
ACL2+Books Manual. The text-based view provided by ACL2-Doc is not ideal for objects like tables, icons, and external links. Emacs users, however, may appreciate the ability to navigate documentation quickly using familiar Emacs movement commands, without leaving Emacs or touching the mouse.

The documentation for ACL2-Doc provides a thorough description, so here we give only a brief introduction. ACL2-Doc is normally loaded automatically by the usual emacs-ac12.el file, or can be loaded directly from emacs/acl2-doc.el. Once loaded, you can invoke it with ' $\mathrm{M}-\mathrm{x}$ acl2-doc' or ' $\mathrm{C}-\mathrm{t}$ g', or by invoking ' $\mathrm{C}-\mathrm{t}$.' to go to a particular topic. The ' $\mathrm{h}$ ' command takes you directly to the help topic, ACL2-Doc, which includes a summary of commands. Single-keystroke commands allow you to follow a link, to move to the next link on the page, to search the manual for a string or a regular expression, to search the index, to see a history of visited topics (which can serve as links), and so on, as well as to download a bleeding-edge copy of the manual from the web. Links are indicated with square brackets, for example, [rewrite], but typing a '<RETURN>' will take you to the topic at the point even if the brackets are missing. The interface is natural for Emacs users, e.g., the go to topic command offers a default and also supports completion.

\subsection{Contributing to the Manual}

The full contents of the ACL2+Books Manual are now part of the Community Books. Everyone in the community is welcome to make improvements to existing documentation and to extend the manual to cover additional libraries. Changes can be discussed and committed at the acl2-books project.

The top-level file for the ACL2+Books Manual is found in doc/top. lisp, and it consists largely of include-book commands. If you contribute new libraries and would like to have their documentation included in the manual, it should usually suffice to include your library into this file. Similarly, if you add documentation to some existing Community Books, you may need to ensure they are included. The documentation for XDOC explains how to build the manual after making your changes.

If you want to clarify or extend an existing topic, it should usually be easy to find the right file. Each of the viewers shows, near the top, the path to the file where the documentation originates. Topics that list "ACL2 Sources" as their origin can be found in system/doc/acl2-doc.lisp, which initially consisted of the documentation that we extracted from a copy of the ACL2 sources in October, 2013.

\section{Custom Manuals}

The ACL2+Books Manual is the most comprehensive publicly available XDOC manual. However, generally speaking, XDOC can easily create a custom manual for any particular set of books.

For organizations that are developing large formal verification projects, this can be especially valuable. While an industrial verification effort might make use of many Community Books, it will also typically include many internally developed books that have not been publicly released, e.g., because they contain proprietary information or are simply not of general interest. As the number of internal books grows, so too does the need for effective documentation.

To document such a project with XDOC, the typical approach is to create a new book, say doc.lisp, that simply includes all of the other books in the project and then invokes the xdoc: : save command. Certifying this book will then produce a new web-based manual. Such an internal manual can include all of the documentation for ACL2, the Community Books you are using, and your internal books. Deploying a web-based manual as part of your automatic build process can make it easy for your team (and organization) to have current documentation covering your entire project. 
A manual produced by xdoc: : save is a new directory that contains a top-level index.html file and other supporting files. These manuals do not require any server-side support, so to deploy one you may only need to upload the directory to your intranet web server or copy it into some shared file system. This works well if all of your users have fast, local intranet connections. However, if your organization includes remote users, the basic manual produced by save may be slow to load: manuals can be tens of megabytes, and by default all of this content is loaded at startup. To avoid this latency, you may wish to deploy a server-supported manual. XDOC includes scripts for converting the manual's contents into a database, and for having a web server access this database to provide topics on an as-needed basis. While this is more work, it allows for manuals that are much more responsive on slow connections.

\section{Automating Documentation and Keeping DRY}

XDOC, like many software documentation tools, takes the approach of embedding the documentation directly into the source code. There are many other ways to document software. For instance, documentation could be kept in a Wiki or put into files such as Microsoft Word documents, LTEX files, HTML files, etc. Documentation could even take the form of traditional, paper books, conference papers, blog articles, presentations, webcasts, and so forth.

These alternatives do have some benefits. Embedding the documentation in the source code limits who can practically edit and contribute to it and, at least to some degree, dictates its form. For instance, good technical writers and graphics artists may have little experience with version control systems and markup languages, and also should perhaps not be directly editing and committing source code.

Even so, there's a lot to be said for putting the documentation right into the code. As a minor benefit, this approach lets developers see the corresponding documentation, albeit in its raw markup form, whenever they are working with the code. It also makes it convenient to update the documentation as the code is changed: you don't also have to find and edit some corresponding Wiki entry or file.

But by far the most important benefit of embedding the documentation into the code is that it can drastically reduce the amount of information that is duplicated between the code and the documentation. This is in keeping with the don't repeat yourself (DRY) principle of software development, popularized by Hunt and Thomas [6]. In practice, we believe this is critical to keeping the documentation up to date as the code base changes. A good documentation tool can avoid this duplication by automatically extracting information from the source code-function signatures, type definitions, how code is organized into modules, and so forth—and making this information available in the manual.

\subsection{Challenges: Modules, Types, and Macros}

In languages like Java and $\mathrm{C}++$, programs are made up of packages, namespaces, classes, etc., that bundle together related functions and structures. Documentation tools for these languages can exploit these scoping constructs to partition the manual into coherent sections automatically, and to automate the generation of sensible navigation pages. They can also make good use of the type declarations in function signatures. Consider some $\mathrm{C}++$ function from LLVM:

Pass* BasicBlockPass: : createPrinterPass

(raw_ostream \&0, const std: string \&Banner) const

$\{\ldots\}$

Even if the programmer doesn't write anything to explain this function, a documentation tool still has a lot to work with. It can link to classes like Pass, raw_ostream, and std: :string. It knows that 
this function should be filed under the BasicBlockPass class. It can link to the parent classes of BasicBlockPass and to the createPrinterPass method in from the superclass that is being overridden. This context may be very useful to a reader who wants to understand what the function does and how it fits into the program.

It is much harder to provide this kind of automatic context and boilerplate in a documentation tool for a dynamically typed language like ACL2. Consider some Lisp function from ACL2's rewriter:

(defun geneqv-refinementp (equiv geneqv wrld) ...)

What does this function return? What kind of object is equiv supposed to be? What is a geneqv? A wrld? You can discover the answers by studying the source code comments and understanding the context, but there is no function signature for a documentation tool to extract. Worse, this function is merely one among many. It's not a member of some class. It has no identifiable relationship to any sort of interface. There is little that a documentation tool can do to provide useful context, here.

Things may not be quite as bad for user-level ACL2 functions. Many of these functions have guards, which are often like type signatures except that they don't explain the return value. Meanwhile, ACL2 books may sometimes contain "type-like" theorems about the return values of functions. Perhaps an ACL2 documentation tool could heuristically identify these theorems. Perhaps it could also use some kind of call-graph analysis or other kinds of hints-file names, packages, common name prefixes, and so on-to group up functionality into related modules. But this does not seem especially promising.

But there's a deeper challenge here. Java and C++ programs have a rich but fixed set of syntactic constructs, like packages, classes, functions, enumerations, and structs. In contrast, as ACL2 users, we often introduce our own custom macros that allow us to quickly define collections of related functions and theorems. Some of these macros are widely applicable, e.g., the std/util library's defaggregate macro introduces something like a struct in C. Other macros are very customized and tailored to specific uses, e.g., the VL library's def-vl-modgen macro is used to define a function that dynamically creates a Verilog module and its supporting modules, according to a certain paradigm. A simple search for "(defmacro def" yields over 500 matches in the Community Books. While exploiting guards and heuristically identifying return-value theorems might allow us to document the output of these macros automatically, the abstraction itself would be lost.

Fortunately, there are good solutions to these problems. In the rest of this section, we'll explore the tools that XDOC provides for structuring books and how macros can be extended to generate high-quality documentation automatically.

\subsection{Automating the Topic Hierarchy}

Even without some kind of module system, it is still very common for closely related definitions to be put together within the same book. In a well-structured project, the way that events are organized into books might often be a good way to organize them in the manual. This can be tedious to implement. The documentation hierarchy is built from the parents of each individual topic, so to accomplish this organization, we may need to set the : parents of each topic in our book to some common parent.

To make this kind of organization simpler to implement, the defxdoc command allows the : parents of a topic to be specified implicitly, using the xdoc: : set-default-parents command. For instance, the oslib library provides various functions for interacting with the operating system. The logical definitions and documentation for most of these functions are located in the same file. Instead of specifying the parents explicitly for each topic, we can simply set a default for the whole file. For instance: 


\subsection{Documentation-Aware Macros}

An important activity in large-scale ACL2 projects is the development of macros to automate common patterns in modeling and proofs. Each use of such a macro might cause several interrelated functions to be defined, various tables to be extended, and many theorems to be proved.

How can we effectively document the functionality created by these macros? A simple and effective answer is: extend the macro itself so that it also generates appropriate defxdoc forms. Minimally, the macro might be augmented with : parents, : short and : long arguments that can be used as the basis for a top-level documentation topic. More sophisticated and automatic documentation may be possible if the macro also gives the user a way to add documentation fragments in appropriate places.

As one example, defaggregate is a macro in the std/util library that can be used to introduce something like a struct in C. It allows documentation to be associated with each field. Here is an example of using this macro, taken from the VL library for working with Verilog:

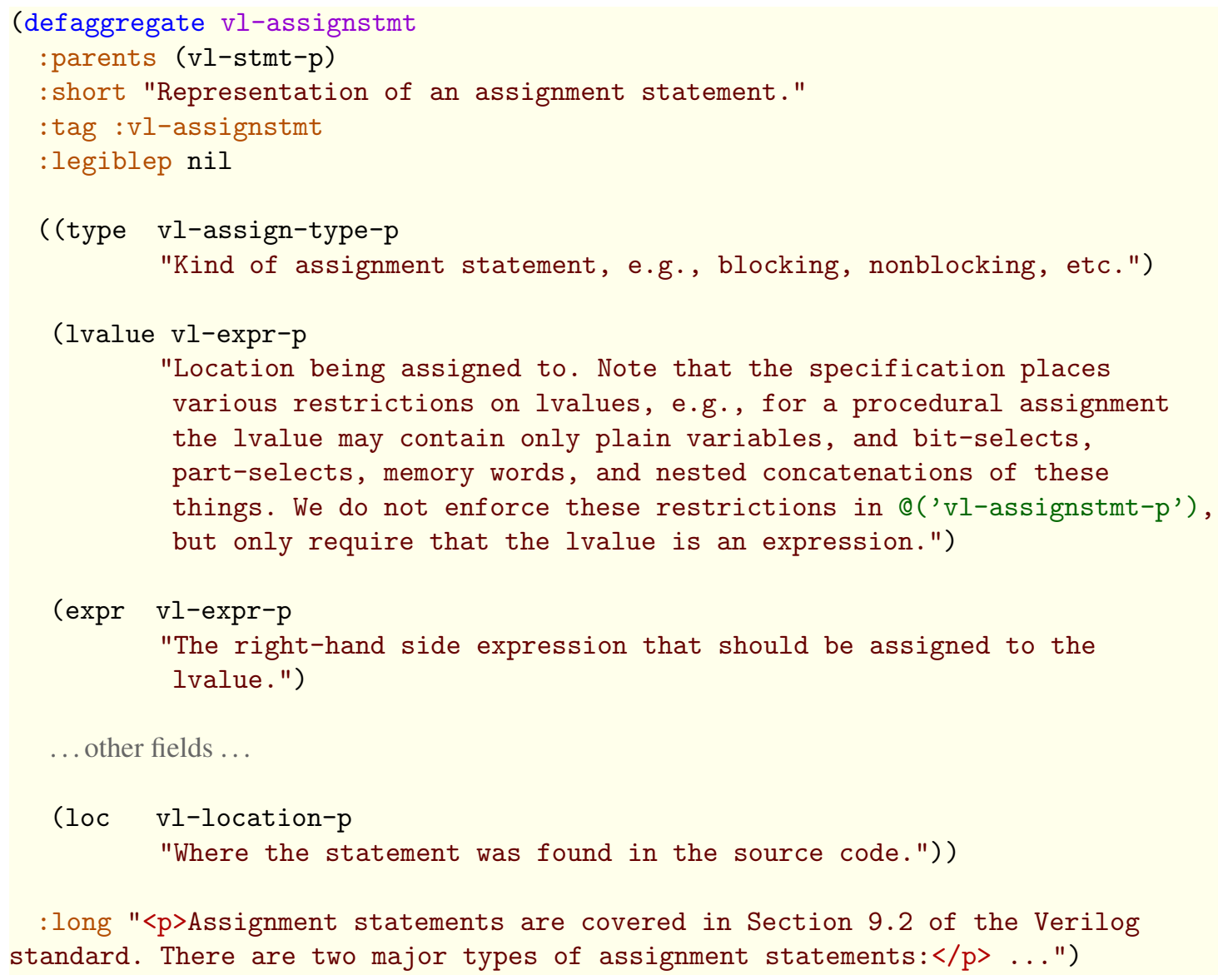

This use of defaggregate produces several ACL2 functions and macros. It creates a recognizer named vl-assignstmt-p, a constructor called make-vl-assignstmt, accessor functions with names like v1-assignstmt->type, and so on. Each of these automatically becomes a corresponding topic in the manual; some examples are shown in Figure 4. These new topics are automatically grouped together with the recognizer being the main topic. Even if we don't add any explicit documentation strings at all, our manuals can at least include boilerplate documentation that, e.g., explains that this structure is produced by defaggregate, shows the fields of the structure and the invariants they must satisfy, gives the syntax for the resulting macros, and so on. When we do provide additional documentation to describe 


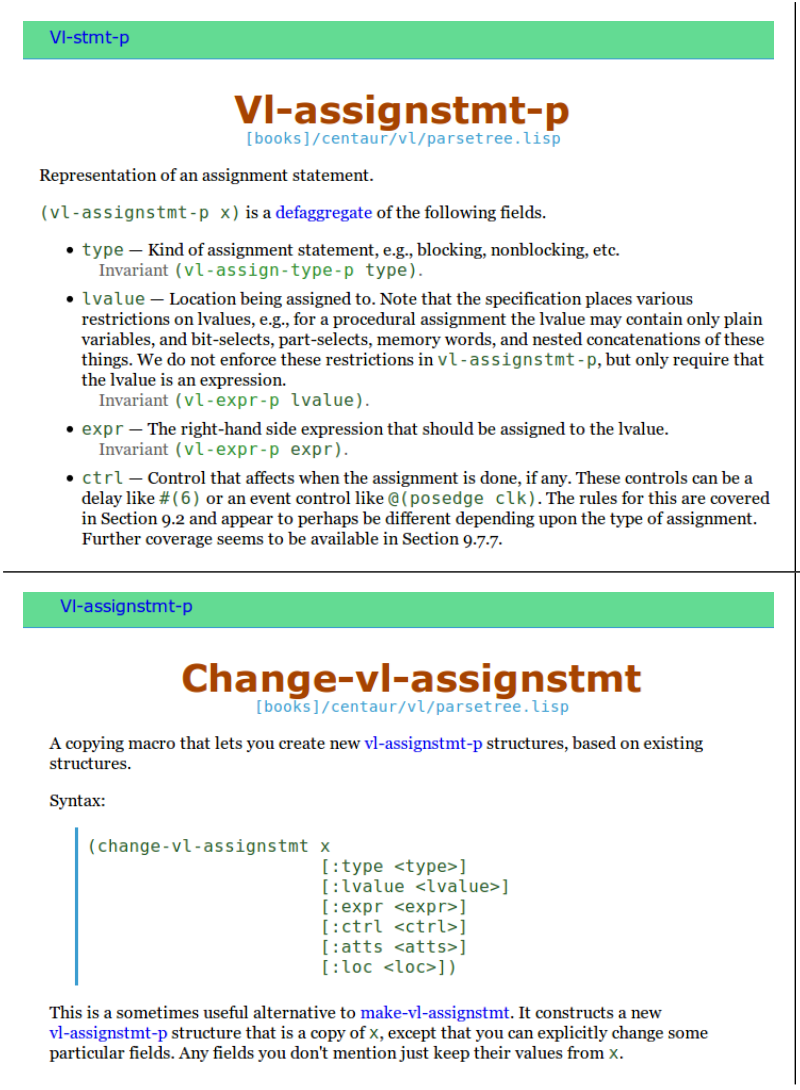

Vl-assignstmt-p
Make-vl-assignstmt
[books]/centaur/vl/parsetree.lisp

Figure 4: Some of the topics generated by defaggregate for vl-assignstmt structures.

the fields of the structure, these explanations are nicely incorporated into the manual.

A really elegant macro is define, also from the std/util library. This macro can be used to introduce, document, and prove theorems about a new function. Here is an example from the bitops library, which we will explain in a moment:

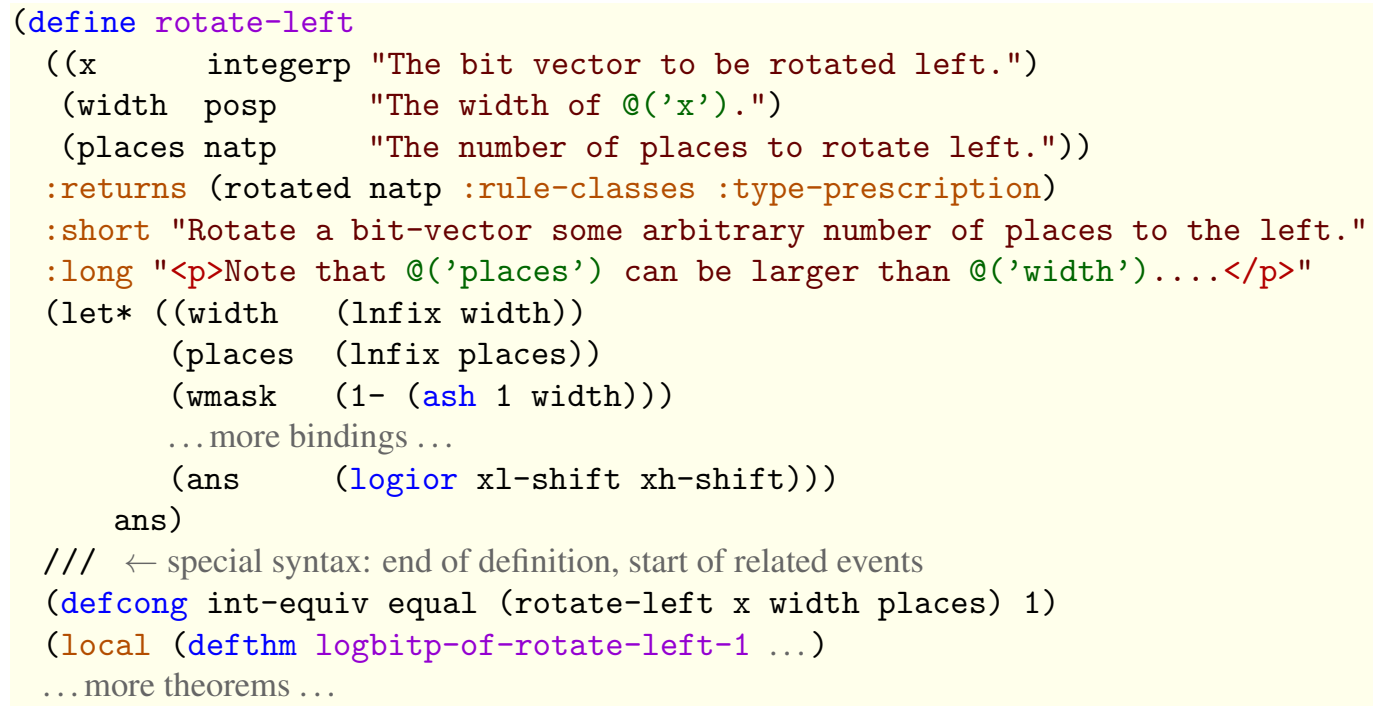






The define macro connects to XDOC in three ways. The most basic is that it can be used as a replacement for defun that simultaneously creates a new topic, e.g., it provides the basic : parents, : short, and : long arguments.

But when documenting a function, you very often want to describe its inputs and return values. To make this more convenient and automatic, define allows the arguments of the functions to be annotated with concise type descriptions (which become guards) and with documentation strings. It also allows the return values of the function to be named and documented, and provides a concise syntax for return-value theorems. This information is merged to create a signature block in the manual that summarizes the function's interface, as shown to the right.

Furthermore, when you introduce a function, it is very common to go on to prove several theorems about it. Using a special /// notation, define provides a built-in area for related events about the new function. These events, along with the definition itself, are actually submitted in a defsection so that they can be automatically

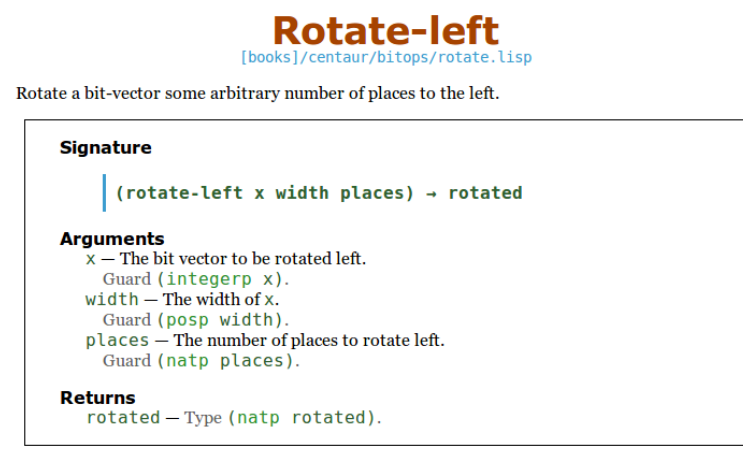

Note that places can be larger than width. We automatically reduce the number of places modulo the width, which makes sense since rotating width-many times is the same as not rotating at all.

Definitions and Theorems

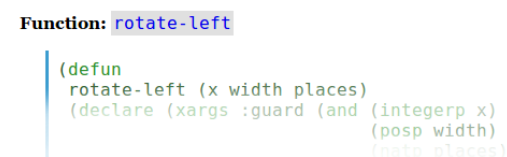

Theorem: logbitp-of-rotate-left-split

(defthm logbitp-of-rotate-left-split

(equal (logbitp $n$ (rotate-left $x$ width places))

(b* ( $(n(n f i x n))$

(width (nfix width))

(places (mod (nfix places) width)))

(cond ( $(>=n$ width) nil)

$((>=n$ places $)$ (logbitp $(-n$ places $) x))$

Theorem: rotate-left-by-zero

$\mid$ (defthm rotate-left-by-zero $\mathbf{x}$ (equal (rotate-left $\mathrm{x}$ width $\theta$ ) included in the documentation.

Putting all of this together, define does a great deal to minimize the amount of redundant information between the code and documentation. While coming up with good ways to integrate documentation strings into macros can require some careful thought, it is not hard to write macros like define. It is mostly a matter of collecting the documentation from the various slots in the macro, and inserting it into a template, e.g., using string concatenation.

\section{Conclusions}

We have developed XDOC, a mature and well-tested tool for documenting formal verification efforts in ACL2. We expect that every organization that is using ACL2 for large projects can benefit from developing an internal XDOC manual.

Note that this paper is not a comprehensive guide to using XDOC. Practical details, such as the available tags in the markup language, the available preprocessor directives, and the specifics of using various commands, can be found in XDOC's documentation about itself; see the XDOC topic in the ACL2+Books Manual. 


\subsection{Related Work}

XDOC treats ACL2 developments more like software than mathematics. It has no support for typesetting mathematical formulas or for explaining the details of how proofs are carried out. In contrast, a great deal of work has been done to allow formal, mechanically checked proofs to be presented as if they were traditional mathematics. For instance, Isabelle can produce nicely typeset $\mathrm{LT}_{\mathrm{E}} \mathrm{X}$ documents [5] from its input files (theories), and interfacing layers like Isar [9] allow the source code to be written in a style that is similar to paper-and-pencil proofs. Other efforts such as the MathWiki project [1] have also focused on develop Wiki systems for formalized mathematics. García-Domínguez [4] et al. have also developed tools for viewing and understanding ACL2 proofs.

XDOC's basic approach — embedding documentation fragments in the code-is used in many popular software documentation generation tools such as Javadoc, Doxygen, and QDoc. These tools have long been used to provide high-quality manuals for large software projects such as the Java platform, QT, KDE, and many others. Similar tools like POD for Perl, RDOC for Ruby, and so on, differ in matters of markup and other details, but are also typically based on documentation comments. The Coq theorem prover has a similar tool, coqdoc, that can produce IATEX and HTML.

Flatt, Barzilay, and Findler [2] have developed an impressive documentation generation system for Racket, called Scribble, which is very different from these comment-based tools. It features a very interesting @ syntax that is closely related to S-expressions but is designed primarily for writing text. This syntax allows for quick transitions between S-expressions and text in documentation. Scribble allows for the documentation to be manipulated by programs, and for the development of custom macros for use in generating documentation.

Knuth has advanced Literate Programming [8] as an alternative to documentation generation systems, where instead of embedding documentation in the code, code is embedded in the documentation. This approach allows the code itself to be structured more like the manual, e.g., definitions might appear in a top-down fashion in the source code file. Gamboa [3] has described an XML-based Literate Programming tool for developing and presenting ACL2 proofs. In this tool, ACL2 source code is extracted from an XML document, perhaps in an out-of-order fashion, so that the document can be arranged for human comprehension, e.g., in a top-down fashion. Separately, the document can be converted into other formats, e.g., web pages.

\subsection{Future Directions}

Although the web-based XDOC viewer has many features, a few known bugs and quirks have been reported in the issue tracker for the Community Books. Its main missing feature is a good searching operation. The web-based viewer can currently search topic names and short strings. Some care has been taken to make this work well, and to feature "important" topics more prominently in the search results. But it would certainly be much better to include the long strings in the search! This seems tricky to implement consistently across both server-supported and local manuals. In the interim, the ACL2-Doc Emacs browser can carry out basic full-text substring and regular-expression searches.

Integrated development environments such as Eclipse and Visual Studio provide extremely useful "intelligent code completion" features for languages like Java. When a programmer who is using this feature begins to type, say, a particular function call, he is automatically shown an unobtrusive reminder of the function's signature and documentation. It would be very exciting to have this capability for ACL2 functions. Perhaps XDOC, with its simple and widely supported markup language, could serve as the data source for this kind of functionality, e.g., in the ACL2 Sedan. 


\subsection{Acknowledgments}

Any manual can only be as good as its content. We have greatly benefited from the many other people who have made substantial contributions to the documentation of ACL2 and the Community Books. Before 2014 (through ACL2 Version 6.4), the authors of ACL2 were responsible for all documentation in the ACL2 User's Manual. They wrote all of it except for certain topics that are typically labeled with their author, and except for the ACL2(r), ACL2(h), and ACL2(p) documentation, which includes contributions from Bob Boyer, Jared Davis, Ruben Gamboa, Warren Hunt, David Rager, and Sol Swords. ACL2's documentation was initially marked up by Laura Lawless. The documentation for the Community Books includes significant contributions from so many authors that we will not try to list them here.

Many other people have made important contributions to XDOC itself. Davis' colleagues at Centaur, including Alan Dunn, Harsh Raju Chamarthi, Sol Swords, and Anna Slobodová, provided extensive beta testing, wrote significant documentation, and gave us helpful feedback and ideas that became important features. Kevin Kramer and Patrick Roberts provided technical support related to hosting XDOC manuals. David Rager was an early adopter and supporter of XDOC, and aside from his many useful suggestions, he has made numerous corrections to many topics.

Warren Hunt was instrumental in pushing us to unify the legacy documentation and XDOC systems. Our conversion of the legacy documentation into XDOC format was made much easier by the legacy documentation system's tools for creating HTML and Emacs Info manuals. These converters were initially developed by Art Flatau and were subsequently improved by Michael Bogomolny and Alan Dunn. J Moore provided useful insights during this conversion process, and also provided extensive beta-testing.

We thank David Rager, Sol Swords, and the workshop reviewers for feedback on drafts of this paper.

This material is based upon work supported by DARPA under Contract No. N66001-10-2-4087, by ForrestHunt, Inc., and by the National Science Foundation under Grant No. CCF-1153558.

\section{References}

[1] Jesse Alama, Kasper Brink, Lionel Mamane \& Josef Urban (2011): Large Formal Wikis: Issues and Solutions. In: Calculemus/MKM '11, pp. 133-148, doi:10.1007/978-3-642-22673-1_10.

[2] Matthew Flatt, Eli Barzilay \& Robert Bruce Findler (2009): Scribble: Closing the Book on Ad Hoc Documentation Tools. In: International Conference on Functional Programming, ACM, pp. 109-120, doi: $10.1145 / 1596550.1596569$.

[3] Ruben Gamboa (2003): Writing Literate Proofs with XML Tools. In: ACL2 '03.

[4] Antonio García-Domínguez, Francisco Palomo-Lozano \& Inmaculada Medina-Bulo (2009): Hypertext Navigation of ACL2 Proofs with XMLEye. In: ACL2 '09, ACM, pp. 47-56, doi:10.1145/1637837.1637845.

[5] Florian Haftmann, Gerwin Klein, Tobias Nipkow \& Norbert Schirmer (2013): $B T_{E} X$ Sugar for Isabelle Documents. Available at http://www.cl.cam.ac.uk/research/hvg/Isabelle/dist/Isabelle2013-2/ doc/sugar.pdf.

[6] Andrew Hunt \& David Thomas (1999): The Pragmatic Programmer. Addison-Wesley.

[7] Matt Kaufmann \& J Strother Moore (1994): Design Goals for ACL2. Technical Report 101, Computational Logic, Inc. Available at http://www.cs.utexas.edu/users/moore/publications/km94.pdf. Also appeared in: Third International School and Symposium on Formal Techniques in Real Time and Fault Tolerant Systems, Kiel, Germany (1994), pp. 92-117.

[8] Donald E Knuth (1984): Literate programming. The Computer Journal 27(2), pp. 97-111, doi:10.1093/comjn1/27.2.97. 
[9] Markus Wenzel (1999): Isar: A Generic Interpretative Approach to Readable Formal Proof Documents. In: TPHOLs '99, LNCS 1690, Springer, pp. 167-183, doi:10.1007/3-540-48256-3_12. 